\title{
Human recombinant alkaline phosphatase: a promising, yet-to-be-tested agent for the treatment sepsis-induced acute kidney injury
}

\author{
Zhongheng Zhang
}

Department of emergency medicine, Sir Run Run Shaw Hospital, Zhejiang University School of Medicine, Hangzhou 310016, China

Correspondence to: Zhongheng Zhang. Department of emergency medicine, Sir Run Run Shaw Hospital, Zhejiang University School of Medicine, No. 3, East Qingchun Road, Hangzhou 310016, China. Email: zh_zhang1984@zju.edu.cn.

Provenance: This is an invited Editorial commissioned by Section Editor Linpei Jia, MD, PhD (Department of Nephrology, Xuanwu Hospital of Capital Medical University, Beijing, China).

Comment on: Pickkers P, Mehta RL, Murray PT, et al. Effect of Human Recombinant Alkaline Phosphatase on 7-Day Creatinine Clearance in Patients With Sepsis-Associated Acute Kidney Injury: A Randomized Clinical Trial. JAMA 2018;320:1998-2009.

Submitted Nov 27, 2018. Accepted for publication Dec 10, 2018.

doi: $10.21037 /$ atm.2018.12.17

View this article at: http://dx.doi.org/10.21037/atm.2018.12.17

Sepsis is defined as an organ dysfunction syndrome caused by the over-activation of inflammatory response $(1,2)$. Kidney is one of the most commonly involved organs in sepsis, and there is a large body of evidence showing that even mild increase in serum creatinine contributes to significantly increased risk of death $(3,4)$. Thus, strenuous efforts have been made to improve renal function during sepsis, including the avoidance of renal toxic agents, treatment with sodium bicarbonate to correct metabolic acidosis (5), and restriction of chloride-rich fluid and starch $(3,6,7)$. However, the risk of acute kidney injury (AKI) is still high in the intensive care unit and a substantial number of patients requires renal replacement therapy to avoid lifethreatening metabolic derangements.

In recent issue of $7 A M A$, Pickkers and colleagues investigated the efficacy and safety of a new agent called human recombinant alkaline phosphatase in the treatment of sepsis-induced AKI (8). Although the primary end point did not reach statistical significance, there is a tread towards improved short-term renal function in the intervention group. Most probably, the beneficial effect of the drug is not as large as being presumed in calculating sample size. Thus, the statistical power of the study is not large enough to detect such a beneficial effect. Patients in intensive care unit (ICU) are usually having coexisting comorbidities and/or acute complications, which may compromise the potential effect of a single intervention. The median sequential organ failure assessment (SOFA) score in the present study is 10 , indicating severe organ dysfunctions beyond the kidney. Other critical conditions such as hypoxemia, hypotension and overactivated inflammatory response contribute significantly to reduced endogenous creatinine clearance (ECC). These factors while distributed unevenly among the study population, also increased the heterogeneity of the study population, further compromising the statistical power of study. Thus, by expanding the sample size, some significantly positive results may be found to support the effectiveness of human recombinant alkaline phosphatase. One limitation of including heterogeneous population is that patients may respond differently to a treatment, and the final effect size is an average of the overall heterogenous patient population. Case-mix or heterogeneity is the curse of critical care researches. Some sophisticated machine learning methods such as model-based recursive partitioning $(9,10)$, latent class analysis with distal outcome can help to identify homogenous subpopulations (11-13). However, these methods require large sample size to have a stable estimate (i.e., the underlying structure of the latent class may be unstable if the sample size is small).

The primary end point of the present study is the area under the ECC curve (AUC) over 7 days after enrollment, which is calculated by the integral of the ECC function over time. There was a difference between treatment and control groups, but statistical significance was not 
reached. The AUC in the treatment group was $55.1 \mathrm{~mL} / \mathrm{min}$ [interquartile range (IQR), 15.0 to $93.9 \mathrm{~mL} / \mathrm{min}$ ] in the $1.6 \mathrm{mg} / \mathrm{kg}$ recombinant alkaline phosphatase group versus $45.6 \mathrm{~mL} / \mathrm{min}$ (IQR, 17.7 to $112.4 \mathrm{~mL} / \mathrm{min}$ ) in the placebo group \{absolute difference, $9.5 \mathrm{~mL} / \mathrm{min}$ [bootstrap $95 \%$ confidence interval (CI), -23.9 to 25.5 ]; $\mathrm{P}=0.47$ \}. In post hoc analysis, the authors found that there was significant difference between the two groups in 21 days [mean difference of $27.6 \mathrm{~mL} / \mathrm{min}$ (95\% CI, 8.7 to $46.6 ; \mathrm{P}=0.004$ )]. Probably, the recombinant alkaline phosphatase may need time to take effect, which is evident in the Figure 2 of the original paper that the two ECC curves deviates more remarkably after day 7 . Thus, there is probably a time-lag before the improvement of renal function can be observed. In such a situation, I propose that a model allowing time-varying effect can be helpful (14). For instance, the distributed lag non-linear model can simultaneously model non-linear exposure-response dependencies and delayed effects. This model has been successfully used in environmental exposure and outcome, in which the effect of an environmental factor does not immediate occurs in time after exposure (15). The recombinant alkaline phosphatase is given in the first 3 days, but the effect cannot be observed immediately and there is a time-lag for the drug to take effect. Thus, a model allowing time lag can be helpful in this clinical scenario. Another statistical model that can help to solve the time-varying effect is the incorporation of time-varying coefficient in Cox regression model (16). While it is a randomized controlled trial that the treated and untreated groups have balanced baseline characteristics (i.e., controlling for baseline covariates in multivariable model is not required), the use of such model with only the treatment variable but allowing its time-varying effect will help to disentangle the effect of recombinant alkaline phosphatase over time. However, all these sophisticated models are only to provide hypothesis-generating results. The final conclusion of the effectiveness of recombinant alkaline phosphatase can be drawn with further RCTs that extend the time window for the assessment of ECC. The sample size should be re-calculated to reach adequate statistical power.

The study is very important in the treatment of AKI because it represents a transition from bench to bedside. Human recombinant alkaline phosphatase has been extensively studied in animal studies that evidence is accumulating supporting its beneficial effect on sepsisinduced AKI $(17,18)$. However, there is a long way to go from animal study to clinical application. The present study is the first well conducted randomized controlled trail aiming to investigate the efficacy and safety of the drug. The authors should be commended for their great effort to find novel agents for the treatment of sepsis-induced AKI.

\section{Acknowledgements}

None.

\section{Footnote}

Conflicts of Interest: The author has no conflicts of interest to declare.

\section{References}

1. Singer M, Deutschman CS, Seymour CW, et al. The Third International Consensus Definitions for Sepsis and Septic Shock (Sepsis-3). JAMA 2016;315:801-10.

2. Zhang Z, Smischney NJ, Zhang H, et al. AME evidence series 001-The Society for Translational Medicine: clinical practice guidelines for diagnosis and early identification of sepsis in the hospital. J Thorac Dis 2016;8:2654-65.

3. Kellum JA, Lameire N; KDIGO AKI Guideline Work Group. Diagnosis, evaluation, and management of acute kidney injury: a KDIGO summary (Part 1). Crit Care 2013;17:204.

4. Saxena A, Meshram SV. Predictors of Mortality in Acute Kidney Injury Patients Admitted to Medicine Intensive Care Unit in a Rural Tertiary Care Hospital. Indian J Crit Care Med 2018;22:231-7.

5. Zhang Z, Zhu C, Mo L, et al. Effectiveness of sodium bicarbonate infusion on mortality in septic patients with metabolic acidosis. Intensive Care Med 2018;44:1888-95.

6. Chawla LS, Bellomo R, Bihorac A, et al. Acute kidney disease and renal recovery: consensus report of the Acute Disease Quality Initiative (ADQI) 16 Workgroup. Nat Rev Nephrol 2017;13:241-57.

7. Zhang Z, Xu X, Fan H, et al. Higher serum chloride concentrations are associated with acute kidney injury in unselected critically ill patients. BMC Nephrol 2013;14:235.

8. Pickkers P, Mehta RL, Murray PT, et al. Effect of Human Recombinant Alkaline Phosphatase on 7-Day Creatinine Clearance in Patients With Sepsis-Associated Acute Kidney Injury: A Randomized Clinical Trial. JAMA 2018;320:1998-2009.

9. Seibold H, Zeileis A, Hothorn T. Model-Based Recursive 
Partitioning for Subgroup Analyses. Int J Biostat 2016;12:45-63.

10. Zeileis A, Hothorn T, Hornik K. Model-Based Recursive Partitioning. J Comput Graph Stat 2008;17:492-514.

11. Collins LM, Lanza ST. Latent Class and Latent Transition Analysis: With Applications in the Social, Behavioral, and Health Sciences. Hoboken: John Wiley \& Sons, 2010.

12. Bakk Z, Tekle FB, Vermunt JK. Estimating the Association between Latent Class Membership and External Variables Using Bias-adjusted Three-step Approaches. Sociol Methodol 2013;43:272-311.

13. Zhang Z, Abarda A, Contractor AA, et al. Exploring heterogeneity in clinical trials with latent class analysis. Ann Transl Med 2018;6:119.

14. Gasparrini A, Armstrong B, Kenward MG. Distributed lag non-linear models. Stat Med 2010;29:2224-34.

Cite this article as: Zhang Z. Human recombinant alkaline phosphatase: a promising, yet-to-be-tested agent for the treatment sepsis-induced acute kidney injury. Ann Transl Med 2018;6(Suppl 2):S124. doi: 10.21037/atm.2018.12.17
15. Zhang Z, Hong Y, Liu N. Association of ambient Particulate matter 2.5 with intensive care unit admission due to pneumonia: a distributed lag non-linear model. Sci Rep 2017;7:8679.

16. Zhang Z, Reinikainen J, Adeleke KA, et al. Time-varying covariates and coefficients in Cox regression models. Ann Transl Med 2018;6:121.

17. Peters E, Ergin B, Kandil A, et al. Effects of a human recombinant alkaline phosphatase on renal hemodynamics, oxygenation and inflammation in two models of acute kidney injury. Toxicol Appl Pharmacol 2016;313:88-96.

18. Peters E, Schirris T, van Asbeck AH, et al. Effects of a human recombinant alkaline phosphatase during impaired mitochondrial function in human renal proximal tubule epithelial cells. Eur J Pharmacol 2017;796:149-57. 